\title{
A FRAMEWORK FOR EXPLORING ETHICAL DILEMMAS IN A FIRST YEAR ENGINEERING COURSE
}

\author{
Carol P. Jaeger and Peter M. Ostafichuk \\ The University of British Columbia \\ carolj@apsc.ubc.ca
}

\begin{abstract}
A module on professionalism and ethics was developed and introduced in a recent redesign of the first year engineering curriculum at The University of British Columbia (UBC). Motivating factors for including this content in first year included providing students with a fuller understanding of the engineering profession, introducing content to support student development in multiple Canadian Engineering Accreditation Board $(C E A B)$ graduate attributes, and providing education and support for students in the responsible use of peer review. Additionally, feedback from senior engineering students indicated that students would benefit from inclusion of professionalism and ethics content earlier in the curriculum. In this paper, the structure and content of the module specifically related to ethics will be described, student feedback for the module will be presented, and key learnings will be discussed.
\end{abstract}

Keywords: codes of ethics, ethical dilemmas, gradual escalation, professionalism, peer review.

\section{INTRODUCTION}

APSC 100, Introduction to Engineering I, was first introduced at UBC in September 2015 [5]. It is an interactive course delivered in a flipped classroom format. Together with the follow on course APSC 101, Introduction to Engineering II, it is intended to provide all students with an introduction to not only specific technical aspects of each engineering discipline, but also to enduring skills such design, decision making, communication skills, professionalism, and ethics. The course is divided into modules, with this two week module being the fourth and final module of the term.

\section{MODULE STRUCTURE, LEARNING GOALS, AND CURRICULUM}

\subsection{Course Flow}

Each week in APSC 100 has the same structure. Students prepare for the week by viewing online content and answering self-test questions. The contact time is structured in a whole-part-whole format, with 50 minute lectures in sections of approximately 200 students being the 'whole' and two hour studios of 60 students being the 'part'. Students are placed in teams and sit with their team in both lectures and studios. The first lecture of the week begins with a short team quiz to ensure that students are well prepared to continue with the learning goals for the week.

This course structure is well suited to the introduction of professional ethics. Online pre-week content was used to introduce new concepts and set the scope for the topics each week and in-class team activities were used to guide students through short example scenarios. Students were then tasked with analyzing larger ethical dilemmas as a team during two-hour weekly studios.

\subsection{Scope}

The two CEAB graduate attributes that were targeted in this module were "professionalism" and "ethics and equity". Further, article 3.4.5.1 of the accreditation criteria and procedures manual [2] mandates that the curriculum for all engineering programs include professional ethics, equity, and law. Prior to the 2015/16 academic year, engineering programs at UBC offered very little content in these topics prior to the final year of study. Surveys of senior year students at UBC revealed that they felt that earlier exposure to these important topics would have been beneficial to their general understanding of the responsibilities of a practicing engineer. Through additional stakeholder consultation it was decided that curriculum content related to law was not appropriate but that first year students should be introduced to the concepts of professionalism and ethics more formally in the first year.

Ethics is a substantial topic in its own right, and to explore the theory of ethical frameworks in depth would take considerably more time than was available. Therefore, it was decided that although an introduction to three notable ethics philosophies would be introduced, the majority of the content would be focused around professional ethics, i.e. the consideration of ethical 
dilemmas likely to occur in an academic setting or in an engineering workplace. Because students have limited personal experience on which to draw in the study of professional ethical dilemmas, a progression of scenarios beginning with personal situations (e.g. peer pressure or the breaking of well understood laws), followed by academically relevant examples (e.g. plagiarism), were used before finally introducing workplace inspired ethical dilemmas.

\subsection{Learning Goals}

The learning goals for this module are summarized in the list below. They have been consolidated for the purposes of this paper. Some goals would have been introduced in stages, adding complexity as the module progressed.

- Describe why professions are regulated

- Describe the risks and advantages of self-regulation

- Describe what Codes of Ethics are and their importance

- Describe the relationship between organizational values, societal values, the law, the Code of Ethics, and personal values

- Explain how personal values, Codes of Ethics, and the law can be used in making defensible decisions in difficult situations

- Apply the UBC Engineering Code of Ethics and/or the APEGBC Code of Ethics to evaluate ethical dilemmas

- Describe and give examples of fair, honest, and respectful feedback in the context of peer and instructor evaluations

- Apply the concept of gradual escalation to identify the correct avenue to report inappropriate behavior

- Describe the concept of 'best practices'

- Give examples of strategies to mitigate risk in the interpretation of values and ethics

\subsection{Codes of Ethics and the Iron Pin Ceremony}

The concept of a regulated profession and the responsibilities of members of such a profession are introduced in this module. Two key reference documents that were introduced are the Association of Professional Engineers and Geoscientists of British Columbia (APEGBC) Code of Ethics [1], and also the UBC Engineering Code of Ethics [6]. The latter is an adaptation of the APEGBC Code of Ethics that is designed to be readily accessible to all students. The tenets are more closely related to academic pursuits and general student conduct. The UBC Engineering Code of Ethics was developed together with the UBC Iron Pin
Ceremony, a new tradition developed by our students to encourage a more inclusive community and to promote the highest standards of student conduct. The Iron Pin Ceremony features short messages of wisdom from industry and university representatives, a group reading of the UBC Engineering Code of Ethics, and the distribution of lapel pins as a keepsake reminder of the students' commitment to the code. For first year engineering students the Iron Pin Ceremony is incorporated into the APSC 100 module on professionalism and ethics.

\subsection{Venn Diagram}

One visual aid that we have employed to help students understand the relationship between personal and societal values, laws, and ethics is a Venn diagram (see Figure 1). The use of the diagram begins with a comparatively simplistic representation of the relationships and is then used to explore the concepts of intent, perception, and appearance of conflict. Use of the diagram in Figure 1 began with crisp lines and clear cut examples of how different actions would be placed. The version with blurred lines as shown was used in subsequent references to the diagram to depict the concept that different perspectives result in different placement of actions on the diagram. The diagram is also used as the focal point of interactive student activities that have yielded interesting results. An example is included in section 4.1.

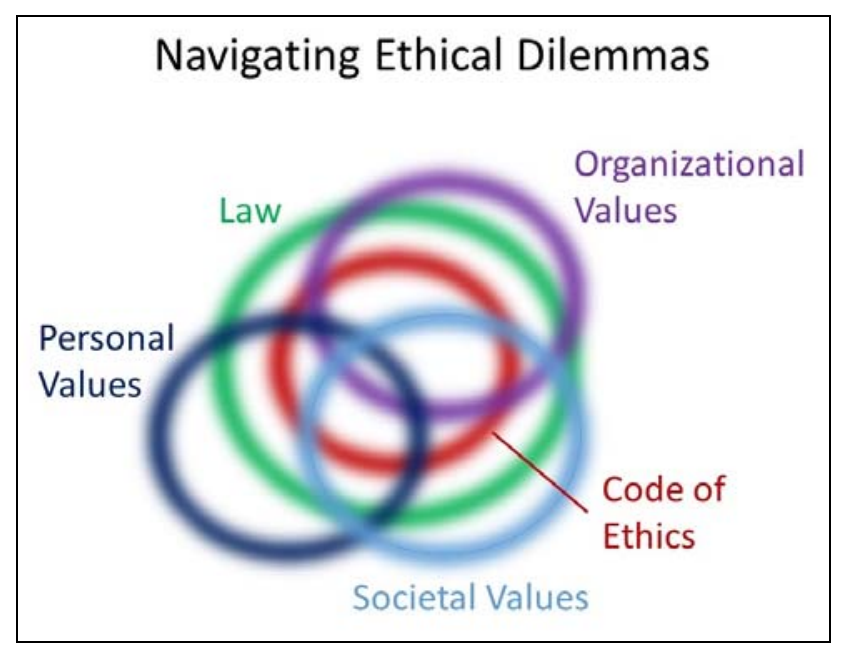

Figure 1: Contextualizing the Code of Ethics

\subsection{Ethical Problem Solving Framework}

By the outset of this module, students are familiar with the engineering design process. In the previous three modules, students have completed two design projects and one decision-making case study that uses a design cycle approach. We chose to extend the methodology of 
using an analogous approach for considering ethical dilemmas by adapting the APSC 100 design cycle, as shown in Figure 2. The decision to use a framework was not to meant to imply that correct decisions could be arrived at by simply following a defined process, but rather that there were similar steps in the design cycle that encourage the student to consider as many stakeholder perspectives and courses of action as possible in the decision making process. A cyclical pattern to the decision making process also aligns well with the introduction of gradual escalation as a best practice for managing difficult situations.

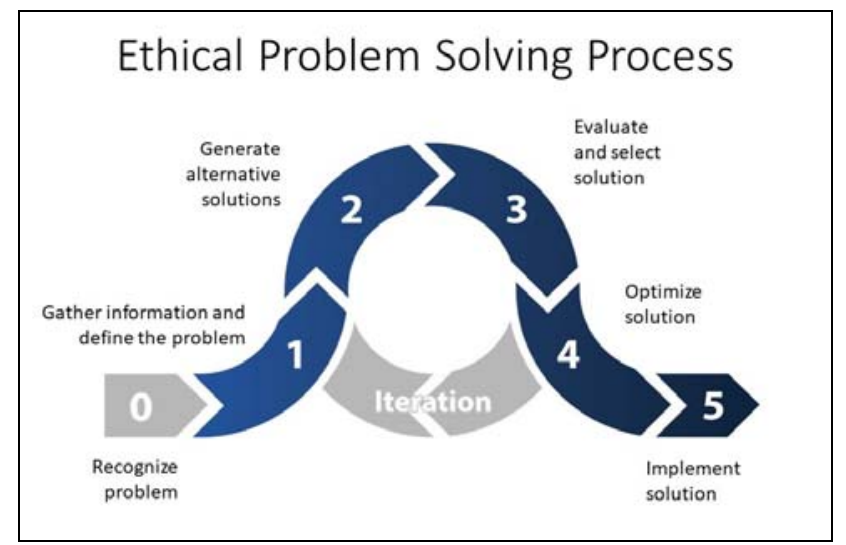

Figure 2: Modified Design Cycle for Application to Ethical Problem Solving

\subsection{Sample Case Studies}

Choosing suitable case studies or scenarios for students to work through was a collaborative process. There is a growing set of open-access online resources available which were reviewed (e.g. [3], [4]). It was concluded that no single resource seemed to contain content that fit the learning goals and course structure asis, and thus new case studies were crafted in-house. Through stakeholder discussions it was identified that choosing scenarios that had relevance for students was critical to student engagement. As part of the scenario creation, colleagues were interviewed about their industrial experience in different engineering disciplines and this information was used to help craft case studies that a student might encounter in early career placements (including co-op positions). The general approach was to create scenarios, or groups of scenarios, that began with a relatable topic for students drawn from personal interactions with peers. Peer pressure, teasing and bullying, respect for commonly recognized laws, drinking and driving, and other similar examples that generally incorporated elements of safety, gradual escalation, and speaking out were representative examples of this category. From these examples, a shift was made to scenarios that were tightly tied to the academic experience, again with the goal of introducing situations that students could relate to and may have already encountered. These included academic misconduct such as plagiarism and cheating on a test, and using unlicensed software to complete a team project. For these examples, the concept of victimless crimes was also introduced. One additional consideration in choosing scenarios was to expose students to various forms of academic and nonacademic misconduct through these examples with the intent that, if a student was not already aware of policies and expectations surrounding student conduct, it would help students to avoid such situations themselves. Finally, examples drawn from industry experiences were introduced. These included the dilemmas of gifts in the workplace and the unenviable position of being tasked with inspecting work on a construction site prior to receiving adequate training.

Each module in this course incorporated some form of communications exercise. In this module the focus was on the giving of respectful feedback and constructive criticism. This aligns with the Code of Ethics tenet related to giving honest and fair professional comment. Students in this course participate in peer evaluations of their team members and this module also coincides with end of term student evaluation of teaching surveys. A discussion around meaningful comments in peer review was part of an in-class activity, and students were given time to complete teaching evaluations as part of a studio session. It should be noted that the activities in this module were limited to written and confidential forms of feedback. Giving and receiving feedback in person is introduced in a module in the follow-on course APSC 101.

\section{OBSERVATIONS AND STUDENT FEEDBACK}

This module has been delivered twice, and feedback from student surveys, focus groups, and observations from course instructors after the first offering was used to make curriculum revisions for the second offering. One key issue that was identified revolved not around the content itself, but around the framing and presentation of the content. The other notable feedback was related to the complexity level of the scenarios used in the modules.

\subsection{Framing the Module}

Students found the transition from a very hands-on engineering design module to a discussion of this very different material a difficult one. By acknowledging this a priori in pre-module content and during in-class discussion, the students seemed to be more comfortable with the change in focus. A related phenomena observed in the first offering was that some students genuinely 
struggled with the notion that they would be involved in an ethical dilemma. In class discussion of how to resolve a dilemma was sometimes met with responses such as "I would never do that, therefore I would never find myself in this situation so there is no dilemma." By prefacing the introduction of the ethical dilemmas with the context that one can be faced with a dilemma without causing it, and by some careful rewording of scenarios to make clear that the person responsible for resolving the dilemma was not the person who created the dilemma, students showed a greater willingness to analyze the dilemmas and work through a decision making process. Personal examples from industrial, academic, and administrative experiences helped to reinforce the fact that one frequently becomes responsible for resolving a situation created by others.

\subsection{Complexity of Scenarios}

Student feedback on the content and the types of scenarios for this module indicated that they wished to see more complex issues as otherwise the activities seemed like a simple application of common sense. Realignment of the content with a more significant introduction in the online pre-week preparation videos has allowed more complex scenarios to be considered in lecture and studio sections.

\section{RESULTS}

In this section highlights of the student response to the 2016 offering of the module will be presented. This includes both observations reported by the instructors and survey results collected upon completion of the module.

\subsection{Observations}

Clear framing of the module coupled with an acknowledgement that the transition from the previous module was difficult in that it took the students from very concrete design exercises to very abstract problem solving exercises helped the students with the transition. Moving more general information and fact-based content to the online material allowed for the introduction of more challenging scenarios in the lectures and studios.

Students can be reluctant to discuss their opinions on the topics of personal values and the overlap with societal values or the law. One very helpful strategy for developing richer conversation in the classroom was to gather anonymous student feedback in survey form as part of the pre-week preparation and present it to students in class. This allowed students to discuss the results without having to share personal opinions. The most interesting example that highlighted the complexity of ethical dilemmas was an activity where students were asked to identify an act or behavior to represent each region in the Venn diagram. The student feedback contained the contradiction that while many students considered the reporting of student misconduct to be both within their personal values and the code of ethics (and therefore within the law), they also considered that reporting the misconduct of a friend to be within the code of ethics but outside of their personal values. The strategy of collecting anonymized feedback as the starting point for discussion, either before the class or during will be continued and perhaps expanded in future offerings as we have noted consistently enhanced participation rates using this strategy.

\subsection{Survey Data}

A voluntary survey was conducted after the module had been completed. Of a class of approximately 800 students, 126 responses were received, representing roughly $15 \%$ of the class.

Students were first asked to rate their confidence in five major topics covered in this module. The response choices were: Very Confident (VC), Confident (C), Somewhat Confident (SC), Not at all Confident (NC), and Unsure (U). The prompts for the questions summarized in Figure 3 were: For each of the major topic areas shown, please indicate how confident you are with that material.

Q1: Identifying and defining ethical dilemmas

Q2: Applying the APSC 100 Ethical Framework (Code of Ethics, law, and personal values) to screen possible actions involving ethical dilemmas

Q3: Applying concepts of gradual escalation, conflict of interest, and/or ethical theories to evaluate ethical dilemmas

Q4: Giving fair and honest professional comment

Q5: Apply strategies to mitigate risk in ethical dilemmas

It was encouraging to see that approximately $75 \%$ of students felt confident or very confident in their understanding of ethical dilemmas and their ability to work through and evaluate a scenario. Further, 75\% of students felt that they would be confident in their ability to apply strategies to mitigate risk. Additionally, students had an option to add free form comments in the survey and while it is difficult to quantify such responses, there were numerous responses that reflected a comprehension of the importance of the material. Several students expressed the sentiment that a mock scenario can never be truly realistic, and that the additional pressures of having to make rapid decisions or safety related decisions would be significantly more difficult. Still, it was evident that having a systematic process to follow and some reference material to use to assess a situation would help in navigating a real ethical dilemma. 


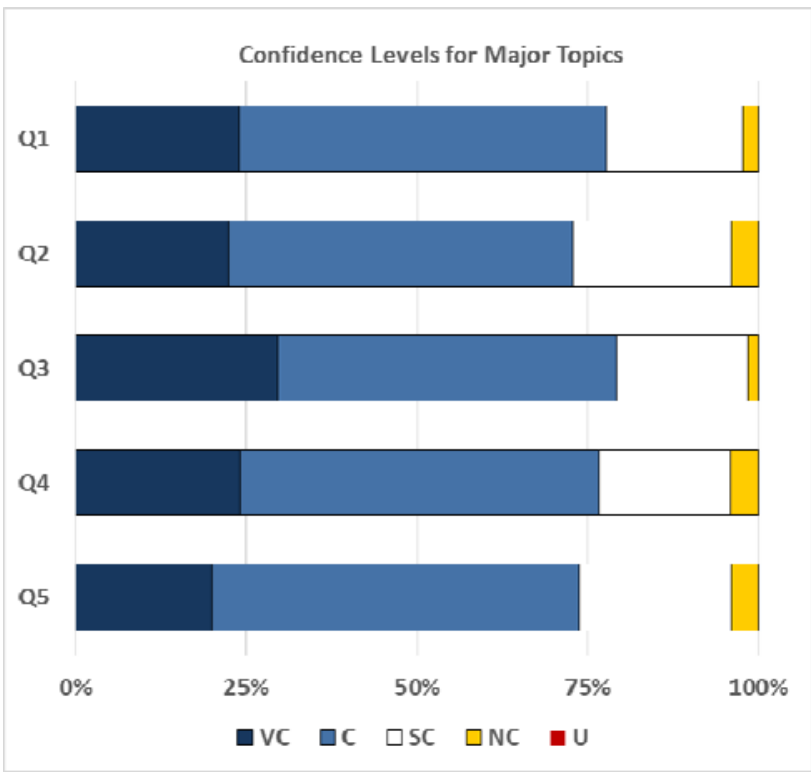

Figure 3: Student assessment of confidence levels for main module topics

Students were then asked some detailed questions about their prior experience with ethics content and their impressions of the types of scenarios presented. The response choices were: Strongly Agree (SA), Mildly Agree (MA), Neutral (N), Mildly Disagree (MD), Strongly Disagree (SD), and Unsure (U). The prompts for the questions summarized in Figure 4 were: Please indicate whether you agree or disagree with each statement below:

Q6: Exploring ethical dilemmas was a new topic for me

Q7: The class and studio scenarios were helpful in allowing me to appreciate the complexities in ethical decision making

Q8: It was helpful to have the opportunity to analyze ethical dilemmas that I was not personally involved in

Q9: I feel as though I have learned useful strategies for managing difficult situations at school and in the workplace

Q10: I felt as though the scenarios presented were realistic and relevant to my future education and career

Q11: I feel as though I would better handle ethical dilemmas that I am presented with now as a result of having completed this module

Q12: I felt engaged with the online and lecture material throughout this module

Q13: The quality of my peer review feedback will be higher as a result of having completed this module

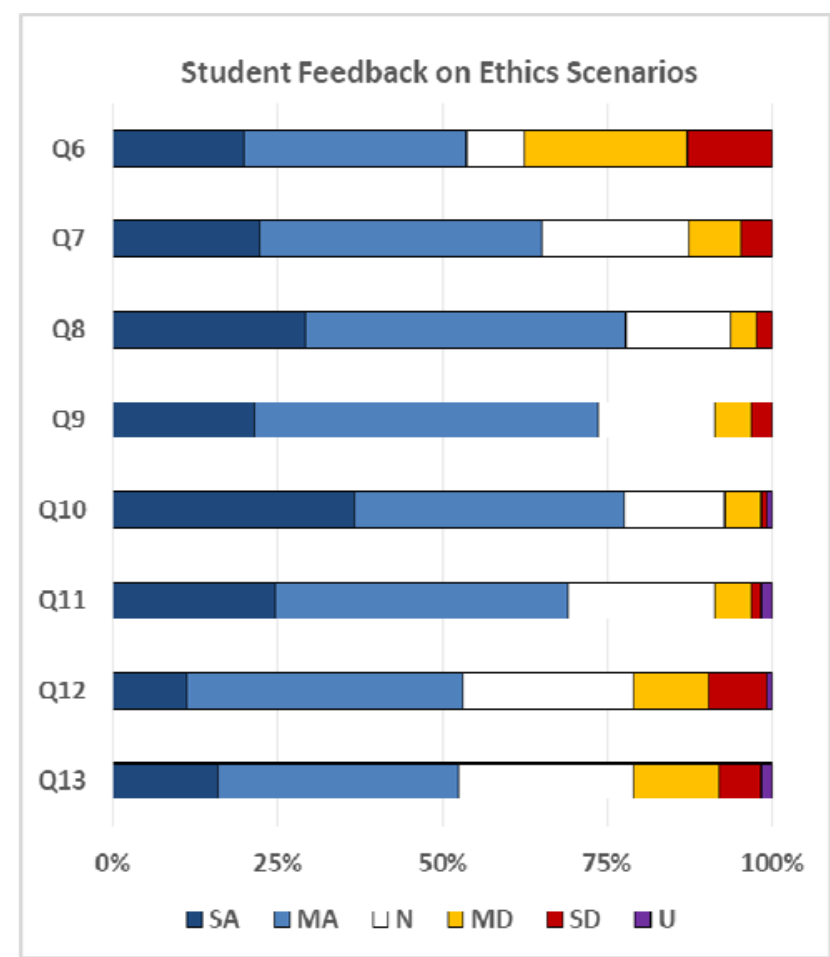

Figure 4: Student response to ethics scenarios

The response to questions were generally positive and validated the feedback from students in the first iteration of the course that the framing of the module and the setup of the case studies important in the acceptance of the content. In particular, the response to question 8 was a strong indication that the introduction of this material is better received if students don't feel as though they have been party to some form of wrongdoing. Additionally, question 10 indicates that the choice of scenarios resonated well as being sufficiently realistic and relevant to the students.

Questions 12 and 13 showed lower numbers of students in general agreement with the associated statements. Question 12 speaks to the level of engagement, which was, not surprisingly, lower than for modules containing more tangible design and build activities. Through the feedback gathered with each offering of the course we will continue to seek ways to engage as many students as possible, but dealing with ethical dilemmas is not generally something one looks forward to either in mock scenarios or in real life. Question 13 combined with the results from question 4 in Figure 3 would suggest that students feel reasonably confident with their ability to give meaningful comments in peer review activities and that the module was at least moderately effective in contributing to or refining these skills. 


\section{CONCLUSIONS AND FUTURE WORK}

In this paper some of the key curriculum elements in a two week module dedicated to professionalism and ethics is described. This module has been designed for a student cohort in their first term of a university program and is intended to give a broad overview of the role and responsibilities of an engineer. Students are exposed to a variety of tools and resources to support them in recognizing, analyzing, and resolving ethical dilemmas in personal, academic, and professional settings. Student response to the content has been generally positive, with students expressing a general understanding of the importance of this material.

In future offerings the scenarios will continue to be developed to make them as engaging as possible for students. The approach of collecting student feedback in anonymous fashion to serve as a basis for in class activities will likely be incorporated more heavily in future. This technique was very effective in soliciting a greater range of student input. Finally, the idea of creating case studies that can evolve in real time based on student input or decisions is being explored as a way to make the in-class experience more engaging. Though such an approach would require a significant amount of advance planning, seeing the connection between actions and consequences has potential to further help students appreciate the complexity of realistic ethical dilemmas.

\section{ACKNOWLEDGEMENTS}

We wish to thank the University of British Columbia Teaching and Learning Enhancement Fund (TLEF) for funding the work to redevelop our first year engineering curriculum.

\section{REFERENCES}

[1] Association of Professional Engineers and Geoscientists of British Columbia (APEGBC) Code of Ethics. Available at https://www.apeg.bc.ca/getmedia/e8d858f5-e1754536-8834-34a383671c13/APEGBC-Code-ofEthics.pdf.aspx

[2] Canadian Engineering Accreditation Board, Engineers Canada, Accreditation Criteria and Procedures, revised February 2017.

[3] Minerva Safety Management Education. Teaching modules available at http://safetymanagementeducation.com/teachingresources/teaching-modules/

[4] National Academy of Engineering, Centre for Engineering Ethics and Society. Open source case studies available at http://www.onlineethics.org

[5] P.M. Ostafichuk, C.P. Jaeger, N. Nakane, S. Nesbit, N. Ellis, J. Sibley, "Redesigning the UBC First Year Introduction to Engineering: Successes and Challenges," in Proc. of CEEA 2016, 8 pp., Halifax, 2016.

[6] UBC Engineering Code of Ethics. Available at $\mathrm{http}: / /$ ubcengineers.ca/eus/traditions/ironpin/ 